\title{
INFLUENCE OF LUBRICATING OIL IMPROVERS ON PERFORMANCE OF CRANKSHAFT SEALS
}

\author{
Piotr Bzura
}

Gdańsk University of Technology, Poland

\begin{abstract}
This paper presents an original method for checking influence of lubricating oil improvers on performance of crankshaft seals of combustion piston engine. Crankshaft seals were tested with the use of a modified friction node of T-02 fourball apparatus in laboratory conditions. The tests were conducted according to a worked-out algorithm. Their results confirmed usefulness of the method for determining ", harmful" performance of sealing systems, which makes it possible to assess operational usability of lubricating oil improvers. On the basis of an analysis of the results a scientific hypothesis saying that lubricating oil quality affects correctness of performance of crankshaft seals of combustion piston engine, was formulated. The hypothesis was verified by means of induction inference and statistical method with taking into account Fisher statistics.
\end{abstract}

Keywords: performance, scientific hypothesis, sealing systems

\section{INTRODUCTION}

During operation of combustion piston engine a part of thermal energy generated by fuel combustion in working space is transformed into mechanical energy. Such form of transformation is called work. Energy transformation efficiency influences mode of operation of a. o. crankshaft bearings . Such operation of an arbitrary slide bearing can be interpreted to be energy transfer over a given time, which makes to-and-fro motion of piston and simultaneously crankshaft rotation possible in given conditions and time t. Wear of all elements of the bearing (i.e. journal, sleeves and lubricating oil - a separating medium) is the main factor affecting slide bearing operation .

Physical and chemical properties of lubricating oil which determine its quality condition operational durability and failure-free operation of crankshaft bearings. In the subjectmatter literature a lack of oil in engine is usually called "oil consumption". However in opinion of this author it is important to distinguish a shortage or loss of oil from real oil consumption. The latter should be meant to be a quantity of oil which goes to combustion chamber and is burned there, whereas a shortage i.e. loss of oil occurs when oil gets off the engine system as a result of leakage.

The shortage (oil loss) depends on operational correctness of crankshaft seals which is affected by quality of improvers. Therefore it is important to investigate the influence.

To perform analysis of influence of improvers on performance of crankshaft seals the friction node of T- 02 fourball apparatus was modified [1]. As a result, it was possible to compare lubricating properties (lubricity) of SAE30 lubricating oil free of improvers with the properties of two samples of the same oil but containing various improvers which make it possible to improve regeneration of boundary layer and consequently to assure boundary lubrication conditions. The so rebuilt boundary layer is characterized by a. o. lowered friction factor, higher durability and resistance to breaking [2].

The presented analysis of results of the tests on the sealing system made it possible to formulate and verify the following scientific hypothesis. 
A sudden break of boundary layer occurs as a result of sudden increase of average value of "harmful" performance of the seal.

Consequences of the hypothesis are the following:

1. An increase of temperature of the unit associated with greater friction,

2. An increase of vibration of the unit associated with direct contact between its elements.

The formulated consequences $\mathrm{K}_{\mathrm{i}}(\mathrm{i}=1,2)$ reveal probabilistic law of wear in the tested crankshaft seal. They are not mutually inconsistent and their logical truth does not involve any doubt. Therefore the condition for consistency of the consequences is satisfied, hence they may be used for empirical testing truthfulness of the presented hypothesis $(\mathrm{H})$, i.e. its verification aimed at either its acceptance or falsification (of course in logical and empirical sense). The verification was done by experimental testing the seal performance and checking truthfulness of the consequences $K_{i}(i=1,2)$. The test confirmed that the consequences really (in fact) occurred. The verification of the hypothesis $\mathrm{H}$ was conducted with taking into account truthfulness of the following syntactic implication $[3,4]$ :

$$
\boldsymbol{H} \Rightarrow \boldsymbol{K}_{i}(i=1,2)
$$

This made it possible to do non-deductive (inductive) inference in accordance with the following scheme [3]:

$$
\left[\boldsymbol{K}_{i}(i=1,2), \boldsymbol{H} \Rightarrow \boldsymbol{K}_{i}(i=1,2] \longmapsto H\right.
$$

Logical interpretation of the inference scheme is as follows : If the empirical test of the consequences $\mathrm{K}_{i}(i=1,2)$ confirmed their truthfulness then, if the implication (1) is also correct, the hypothesis $\mathrm{H}$ is also correct and can be accepted. The inductive inference conducted according to the scheme (2) is called reductive inference. Such inference - like any else belonging to this group of inference - does not lead to certain conclusions but only to probable ones [5]. The tests performed with taking into account the relations (1) and (2) confirmed truthfulness of the formulated hypothesis.

The correctness of the hypothesis - as it was considered probable - was additionally verified by means of Fisher statistics [6].

Results of the verification are given in Sec. 5 of this paper.

The tests in question were carried out on the engine crankshaft standard sealing system whose schematic diagram is presented in Fig. 1.

\section{CRANKSHAFT SEALING SYSTEM}

The sealing system (seal) of crankshaft was consisted of elements which prevent or limit lubricating oil escaping from working space of the seal into the environment.

The aim of sealing technique is to form structural features of the seal itself or the entire sealing unit in such way as to cause it working with a given tightness degree in given operational conditions.

Today, among the seals in rotational motion the most commonly applied design is the so called „simmering” (Fig.1). It is consisted of the metal casing (5) in which a dynamic sealing edge is placed in the form of the lip (3) made usually of an elastomer and pressed towards surface of the shaft (2) by means of the tension spring (4).

The seal operates on lubricating oil boundary layer of abt. $1 \mu \mathrm{m}$ in thickness, that is assured owing to balance between the radial forces $\left(\mathrm{P}_{\mathrm{r}}\right)$ exerted by the tension spring (3), as well as rubber elastomer (2) and the elastohydrodynamic force $\left(\mathrm{P}_{\mathrm{h}}\right)$ resulting from rotational motion of the shaft against the elastomer rubber lip separated from the shaft surface by the oil film (1) [7].

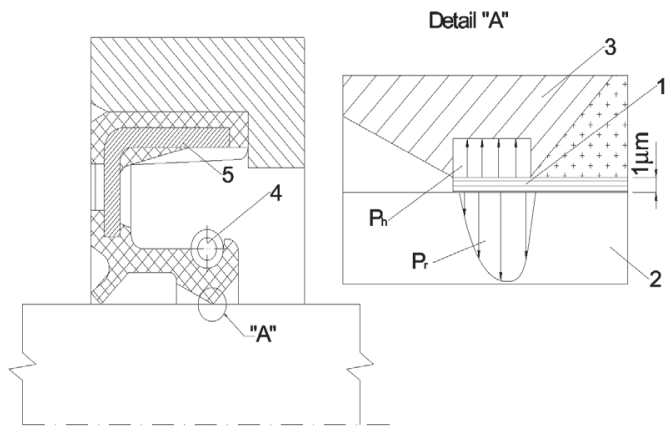

Fig.1. Schematic diagram of the rotating shaft sealing system: 1 - oil film, 2 - crankshaft, 3 - rubber lip, 4 - tension spring, 5 - metal casing, $P_{h}$ - elastohydrodynamic force, $P_{r}$ - radial force

Dynamic seal cannot be always perfectly tight. Permanent tightness may be assured only when a determined amount of oil is able to escape outside and lubricate this way the seal and prevent it against wear and possible failure.

Perfect tightness (in physical sense) is not possible to occur, therefore a technical tightness which takes place when "leakage" is reduced to a level which does not cause any negative consequences for both the environment and device itself, is defined.

Possible assurance of required tightness may be defined as a "harmful" work of the seal, i.e. that which causes a minimum (but necessary) energy dissipation. Such operation of the sealing system intensifies along with increasing wear of one of the crucial elements of crankshaft seal and it may be presented in the following form as the "harmful" performance $[3,4,8]$ :

where:

$$
D_{S}=L_{S} \cdot \tau
$$

$\mathrm{D}_{\mathrm{S}}$ - "harmful" performance of sealing system,

$\tau$ - period of one crankshaft rotation,

$\mathrm{L}_{\mathrm{S}}$ - "harmful” work done during one crankshaft rotation, i.e:

where:

$$
L_{s}=2 \cdot \pi \cdot P_{r} \cdot r \cdot \mu
$$

$\mathrm{P}_{\mathrm{r}}$ - radial load $[\mathrm{N}]$;

$\mu$ - friction factor [-];

$\mathrm{r}-$ shaft radius $[\mathrm{m}]$. 
For purposes of assessment of tightness degree ("harmful" performance) of sealing system the following states are distinguished :

I. Full serviceability state - technical tightness (full),

II. Partial serviceability state - „sweating seal ” or oil leaking through seal,

III. Unserviceability state -leakage.

In determining whether a given sealing system is in the serviceability state (technical tightness) or in the unserviceability state (leakage) a permissible degree of “ harmful" performance $\left(\mathrm{D}_{\mathrm{S}(\mathrm{dop})}\right)$ should be selected as that which causes the highest necessary energy dissipation which does not yet disturb regeneration of the boundary layer formed on friction surfaces of the sealing system. If quantity of the "harmful" performance $\mathrm{D}_{\mathrm{S}} \leq \mathrm{D}_{\mathrm{S}(\mathrm{dop})}$ then the system in question is in the state of full serviceability (technically tight), and, when quantity of the "harmful" performance $\mathrm{D}_{\mathrm{s}}>\mathrm{D}_{\mathrm{s} \text { (dop) }}$ then the system in question goes either to the state of partial serviceability or unserviceability.

Preliminary tests of the so defined performance of the sealing system were conducted on the test stand shown in Fig. 2 and 3.

\section{LABORATORY STAND FOR TESTING "HARMFUL" PERFORMANCE OF SEAL}

The main element of the test stand (Fig.2) was a friction unit formed of four balls of $12,7 \mathrm{~mm}$ in diameter immersed in the tested lubricating medium. The three balls (4) are placed in a lower holder (a sump made of a bearing steel of 62,7 HRC hardness) and considered to represent a crankshaft. Into the sump the tested lubricating medium (a volume of $8 \pm 2 \mathrm{~cm}^{3}$ ) is poured. The fourth ball (3) fixed in the upper holder (a modification of friction unit) is made of rubber of hardness in the range of $15-80^{\circ} \mathrm{sh}$, and considered to represent physical model of the crankshaft seal ring. The balls located in the sump (5) are pressed with the force P produced by the weight (8) against the ball fixed in the upper holder. The rubber ball in the holder (2) is loaded by the reaction force $\mathrm{R}$ equal to the force $\mathrm{P}$ exerted onto the steel balls.

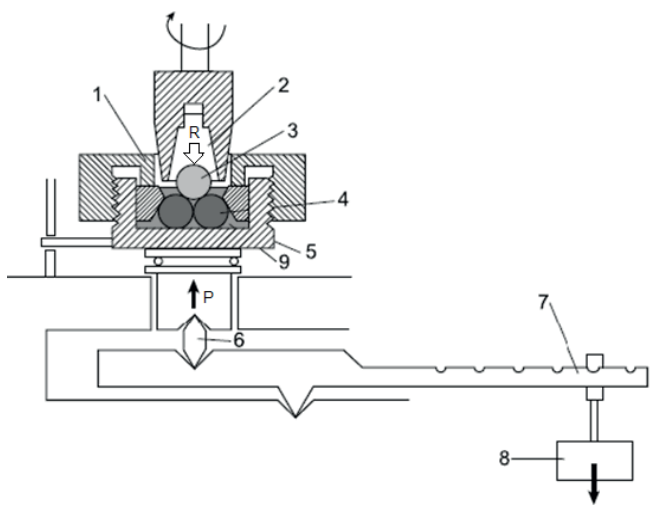

Fig. 2. Kinematic scheme of the four-ball apparatus : 1 - casing to fix lower balls, 2 -upper ball holder, 3 - upper rubber ball (rotating), 4 - lower balls (motionless), 5 - sump with tested oil, 6-prism, 7 -lever, 8 -weights, 9 - tested oil, $P$ - force applied to steel balls placed in sump, $R$ - reaction force [1]
As results from the distribution of forces over the contact of friction pairs according to the standard [9] Fig. 3, shaft radius may be taken equal to that of the circle built by contact points of the balls (3) in friction node, and the acting forces may be determined as follows: $\mathrm{P} \equiv 3 \cdot \mathrm{P}_{\mathrm{r}}$.

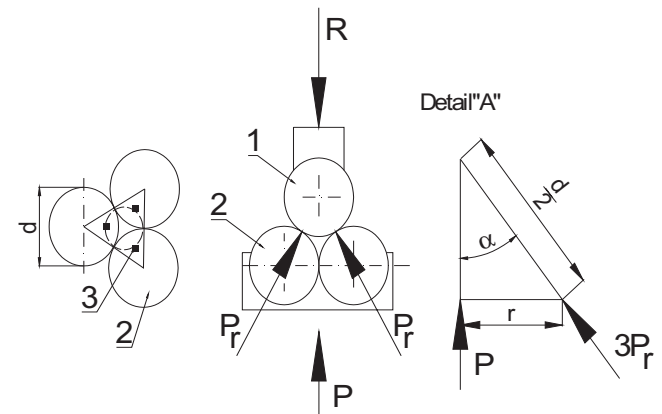

Fig. 3. Distribution of forces in friction node of four-ball apparatus : 1-upper rubber ball, 2 - lower steel balls, 3 - contact points, $d$ - ball diameter equal to $12,7 \mathrm{~mm}, r$-radius of the circle formed by contact points, $a=35,2644^{\circ}$ - angle which determines contact points between the upper (rubber) ball and the lower (steel) balls; $P$ - load applied to steel balls placed in the sump, $R$ - eaction force, $P_{r}$ - radial load.

Based on the detail "A" in Fig. 3, the radius $\mathrm{r}=\frac{d}{2} \cdot \sin \alpha$ and the radial load $P_{r}=\frac{P}{3 \cdot \cos \alpha}$ was calculated.

During the tests the measuring system of the apparatus makes it possible to determine friction factor of the tribological system presented in Fig. 2 and 3. The so determined radius $r$, force $\mathrm{P}_{\mathrm{r}}$ and friction factor $\mu$ were necessary for calculation of the work $\mathrm{L}_{\mathrm{s}}$ (4), that made it possible to determine performance of the crankshaft sealing system in compliance with the formula (3).

\section{DETERMINATION OF PERFORMANCE OF CRANKSHAFT SEALING SYSTEM}

Crucial impact onto performance of crankshaft sealing system results from : crankshaft rotational speed, friction node temperature, friction in seal-shaft pair (depending on the oil viscosity $\eta[\mathrm{mPa} \cdot \mathrm{s}]$ ), power losses (depending on drag torque and shaft rotational speed), oil pressure, materials of which crankshaft and seal is made, principal dimensions of sealing system etc.

For the testing of sealing action with the use of the modified friction node of T-02 four-ball apparatus the constant oil temperature of $20^{\circ} \mathrm{C}$ and constant load of $150 \mathrm{~N}$ was assumed and variable rotational speed of the upper ball holder was selected as the control parameter of influence of lubricating oil improvers on the action.

The three following clean lubricating oils whose dynamic viscosity at $20^{\circ} \mathrm{C}$ was determined by means of DV-1P apparatus produced by Anton Paar $\mathrm{GmbH}$, were tested:

1. SAE30 lubricating oil ( $\eta=240 \mathrm{mPas})-$ marked A.

2. SAE30 lubricating oil containing improving liquid $(\eta=350 \mathrm{mPas})-$ marked B.

3. SAE30 lubricating oil containing improving powder $(\eta=375 \mathrm{mPas})-$ marked $\mathrm{C}$. 
Series of measurements were done on the test stand in accordance of the algorithm shown in Fig. 4. The start-up instant when a sudden rise of " harmful" work occurs giving evidence for breaking the lubricating oil boundary layer, was assumed the permissible degree of the" harmful" performance $\left(\mathrm{D}_{\mathrm{S}(\mathrm{dop})}\right)$.

The tests of each model were repeated four times. Average values of the test results are contained in Tab. 1,2 and 3 and illustrated in Fig. 5, 6 and 7.

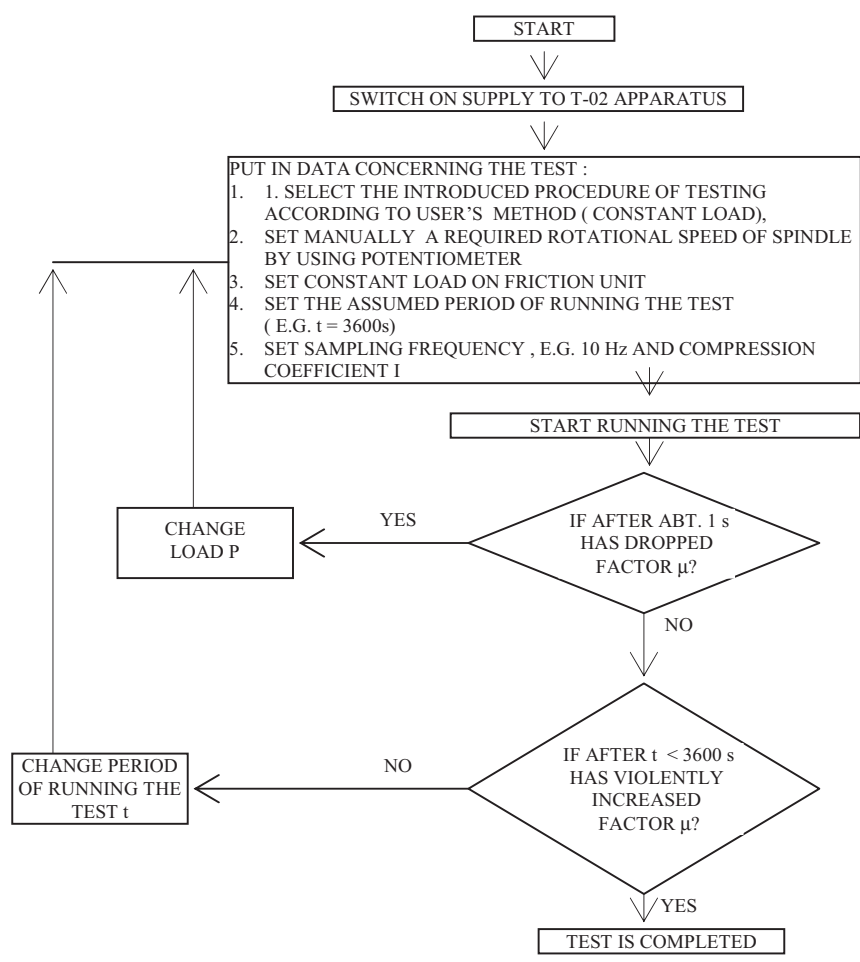

Fig.4. Algorithm of the tests carried out on the measurement stand

Results of the tests according to the worked - out algorithm (Fig. 4) are presented for the rotational speed $\mathrm{n}=312 \mathrm{rpm}$ in Tab. 1 which contains values of the "harmful" performance determined in compliance with the formula (3) for given measurement duration times. And, the performance in function of measurement duration time is illustrated in Fig. 5.

Tab. 1. "Harmful" performance of the seal ring for the rotational speed of $312 \mathrm{rpm}$

\begin{tabular}{|c|c|c|c|}
\hline \multirow{2}{*}{$\begin{array}{c}\text { Measurement duration time } \\
{[\mathrm{s}]}\end{array}$} & \multicolumn{3}{|c|}{ Harmful" performance [mJ-s] } \\
\cline { 2 - 4 } & Mark A & Mark B & Mark C \\
\hline 0,19 & 82,54 & 65,85 & 77,90 \\
\hline 10,00 & 40,81 & 38,02 & 38,02 \\
\hline 20,00 & 35,24 & 36,17 & 41,73 \\
\hline 50,00 & 28,75 & 38,95 & 37,10 \\
\hline 80,00 & 38,95 & 38,95 & 40,81 \\
\hline
\end{tabular}

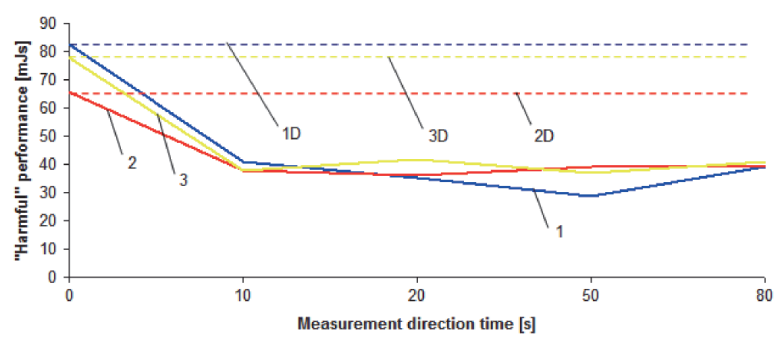

Fig. 5 Comparison of seal ring performance for various lubricating oils at the rotational speed of $312 \mathrm{rpm}$ under the load of 61,2 N: 1 - mark $A$

$1 D$ - permissible performance - mark $A ; 2$ - mark $B ; 2 D$ - permissible performance-mark $B ; 3$ - mark $C ; 3 D$-permissible performance-mark $C$

At the set load and rotational speed of $312 \mathrm{rpm}$ the permissible level of "harmful" performance $\mathrm{D}_{\mathrm{S}(\mathrm{dop})}$ was not exceeded in any case - it means that the lubricating oil boundary layer was regenerated in all the cases.

Similar tests were conducted for the rotational speed of $500 \mathrm{rpm}$. Their results are contained in Tab. 2 and illustrated in functional form in Fig. 6.

Tab. 2. "Harmful" performance of the seal ring for the rotational speed of $500 \mathrm{rpm}$

\begin{tabular}{|c|c|c|c|}
\hline \multirow{2}{*}{$\begin{array}{c}\text { Measurement duration } \\
\text { time [s] }\end{array}$} & \multicolumn{3}{|c|}{ "Harmful” performance [mJ·s] } \\
\cline { 2 - 4 } & Mark A & Mark B & Mark C \\
\hline 0,12 & 45,10 & 40,42 & 48,03 \\
\hline 10,00 & 22,26 & 22,84 & 26,36 \\
\hline 20,00 & 20,50 & 22,84 & 25,19 \\
\hline 50,00 & 21,09 & 22,84 & 25,77 \\
\hline 100,00 & 22,84 & 22,26 & 25,19 \\
\hline 120,00 & 55,06 & 23,43 & 26,36 \\
\hline 150,00 & & 24,60 & 28,11 \\
\hline 200,00 & & 19,33 & 28,70 \\
\hline 201,00 & & 20,50 & 28,11 \\
\hline 209,00 & & 21,09 & 50,24 \\
\hline 280,00 & & 17,57 & \\
\hline
\end{tabular}

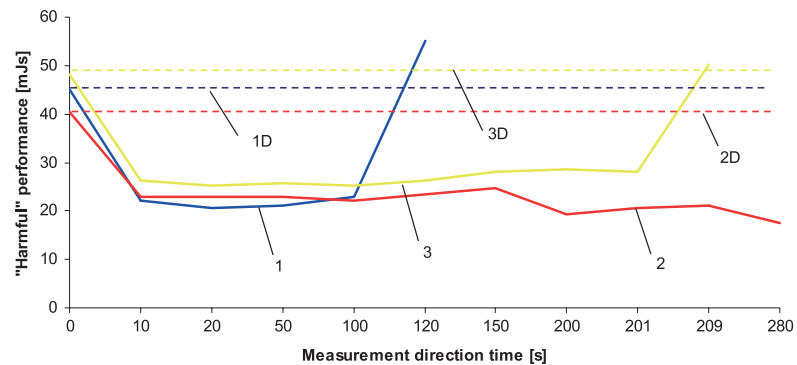

Fig.6. Comparison of seal ring performance for various lubricating oils at the rotational speed of $500 \mathrm{rpm}$ under the load of 61,2 N: 1- mark $A, 1 D$ permissible performance - mark $A ; 2$ - mark $B ; 2 D$ - permissible performance - mark B; 3 - mark $C ; 3 D$ - permissible performance - mark $C$

At the set load and the rotational speed of $500 \mathrm{rpm}$ only SAE 30 oil with improving liquid prevented against exceeding 
the permissible level of 'harmful" performance $\mathrm{D}_{\mathrm{S}(\mathrm{dop})}$ and the boundary layer was regenerated.

And, the tests carried out at the rotational speed $n=1450$ rpm made it possible to achieve the results presented in Tab. 3. Fig. 7 shows the "harmful" performance in function of measurement duration time $\left(D_{S}=f(\tau)\right)$.

Tab. 3. "Harmful" performance of the seal ring for the rotational speed of $1450 \mathrm{rpm}$

\begin{tabular}{|c|c|c|c|}
\hline \multirow{2}{*}{$\begin{array}{c}\text { Measurement duration time } \\
{[\mathrm{s}]}\end{array}$} & \multicolumn{3}{|c|}{ "Harmful” performance [mJ·s] } \\
\cline { 2 - 4 } & Mark A & Mark B & Mark C \\
\hline 0,04 & 14,06 & 15,03 & 12,50 \\
\hline 10,00 & 17,38 & 3,71 & 5,66 \\
\hline 20,00 & & 4,49 & 6,64 \\
\hline 50,00 & & 5,86 & 4,10 \\
\hline 60,00 & & 22,26 & 4,30 \\
\hline 70,00 & & & 6,05 \\
\hline
\end{tabular}

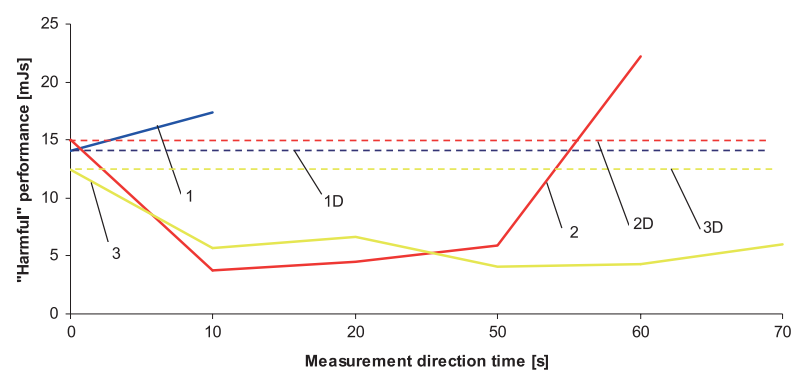

Fig.7. Comparison of seal ring performance for various lubricating oils at the rotational speed of $1450 \mathrm{rpm}$ under the load of 61,2 N: 1 - mark $A$, $1 D$-permissible performance - mark A; 2-mark B; $2 D$-permissible performance - mark $B ; 3$ - mark $C ; 3 D$ - permissible performance - mark $C$

In the third series of the tests at the set load and the rotational speed $n=1450$ rpm only SAE 30 lubricating oil with improving powder prevented against the exceeding of the permissible level of "harmful" performance $\mathrm{D}_{\mathrm{S}(\mathrm{dop})}$.

The tests confirmed that the consequences $\mathrm{K}_{\mathrm{i}}(\mathrm{i}=1,2)$ do occur during operation of the seal. Therefore on the basis of the inference rule (2) it may be stated that the hypothesis is correct. However its truthfulness is not certain but only probable. For this reason the statistical method (statistical inference) was used for its further verification.

\section{VERIFICATION OF THE TEST HYPOTHESIS}

The statistical testing of the scientific hypothesis which states that: sudden break of boundary layer occurred because a sudden rise in average "harmful" performance of seal was revealed, was carried out in the three stages [6]:

1. It was demonstrated that the "harmful" performance of seal is a quantity which may be considered a random variable. The hypothesis that quality of lubricating oil improvers does not affect the "harmful" performance of sealing system was assumed to serve as the zero hypothesis $\left(\mathrm{H}_{\mathrm{O}}\right)$. The hypothesis that quality of lubricating oil improvers affects the "harmful" performance of sealing system was assumed to serve as the alternative hypothesis $\left(\mathrm{H}_{\mathrm{a}}\right)$.

2. From the Kolmogorov-Smirnov goodness-of-fit test for tests of the sample size $\mathrm{n}<100$ and the confidence interval $b=95 \%$ it results that normal distribution is the function which describes mode of assigning probabilities to particular values of random variable.

3. After verification of the hypotheses $\mathrm{H}_{\mathrm{O}}$ and $\mathrm{H}_{\mathrm{a}}$ by means of Fisher statistics for the significance level $\mathrm{a}=0,05$ the hypothesis $\left(\mathrm{H}_{\mathrm{O}}\right)$ was rejected and the alternative hypothesis $\left(\mathrm{H}_{\mathrm{a}}\right)$ was approved.

\section{FINAL REMARKS AND CONCLUSIONS}

The physical model of seal ring should be considered preliminary but that which makes it possible to work out a more adequate physical model of the tested seal ring because it allowed to confirm truthfulness of the hypothesis formulated in the tests. Obviously, the final confirmation of truthfulness of the hypothesis should result from the testing of an original crankshaft sealing system, which is planned in the near future.

From the presented results of the tests arises that:

1. The upper rubber ball of the friction unit may be considered a sealing lip;

2. The three balls inside the cuplike sump were assumed to represent crankshaft;

3. After start-up and the first rotation of the shaft, the permissible level of "harmful" performance was calculated ; its exceeding had to demonstrate that the seal passed to the state of partial serviceability or complete unserviceability;

4. In the instant of losing capability of regenerating boundary layer between the edge of the upper rubber ball (sealing lip) and the edges of the balls inside the oil sump (crankshaft) a violent rise of friction factor (increase in temperature of the set of balls) occurs and consequently results in a failure of the upper ball (a rise in vibration level of the set of balls and separation of a rubber part off the upper ball);

5. Improvers added to SAE30 lubricating oil increased its lubricity (capability of regenerating its boundary layer) which is not strictly equivalent to dynamic viscosity. The sample marked B of a lower dynamic viscosity than that of the sample marked $\mathrm{C}$ demonstrated a higher lubricity for $\mathrm{n}=500 \mathrm{rpm}$.

As results from the tests, the interpretation (proposed by this author) of operation of the sealing system tested in T-02 four-ball apparatus may be used for the determining of usability of improvers added to lubricating oils in service of the systems. The achieved results of the tests allow to state that the test method, proposed by this author, for the testing of influence of lubricating oil improvers on crankshaft operation will be also useful for carrying out similar tests on other sealing systems. 
1. Michalczewski R., Szczerek M., Tuszyński W., Wulczyński J.: A four-ball machine for testing anti-wear, extremepressure properties, and surface fatigue life with a possibility to increase the lubricant temperature. Tribologia 1/2009, pp.113-127

2. Laber A.: Modifying the operating conditions of friction pairs with solid lubricant based additives. Tribologia 5/2011, pp.137-145

3. Girtler J.: A method for evaluating the performance of a marine piston internal combustion engine used as the main engine on a ship during its voyage in different sailing conditions. Polish Maritime Research, No. 4/2010, vol.17

4. Girtler J.: Method of evaluation of lubricating ability of lube oils, diesel oils and heavy fuel oils in energetistic formulation. Journal of POLISH CIMEEAC, Vol. 3, No. 1, 2008

5. Girtler J.: Physical aspect of application and usefulness of semi-Markovian processes for modeling the processes occurring in operational phase of technical objects. Polish Maritime Research, No. 3/2004, vol. 11, pp. 25-30.

6. Bzura P., Dziedzic A.: Analysis of influence of lubricating oil quantity and quality on operational correctness of crankshaft slide bearings (in Polish. Publishing House of Maritime Academy of Szczecin, Szczecin 2016, pp.30-36.

7. Beek A. V.: Machine lifetime performance and reliability, TU Delft, Delft, 2004.

8. Rudnicki J.: Evaluation of compression realization in diesel engine based on performance indicator changes. Journal of Polish CIMEEAC, Vol. 7, No. 1, Gdańsk 2012

9. Designation: ASTM D5183 - 05(2016) Standard Test Method for Determination of the Coefficient of Friction of Lubricants Using the Four-Ball Wear Test Machine
Piotr Bzura

Gdańsk University of Technology 11/12 Narutowicza St. 80 - 233 Gdańsk

Poland 